\title{
Furstenberg families and chaos on uniform limit maps
}

\author{
Risong Li*, Yu Zhao, Hongqing Wang \\ School of Science, Guangdong Ocean University, Zhanjiang, Guangdong, 524025, People's Republic of China. \\ Communicated by F. Vetro
}

\begin{abstract}
Let $\left(f_{n}\right)$ be a given sequence of continuous selfmaps of a compact metric space $X$ which converges uniformly to a continuous selfmap $f$ of a compact metric space $X$, and let $\mathcal{F}, \mathcal{F}_{1}$, and $\mathcal{F}_{2}$ be given Furstenberg families. In this paper, we obtain an equivalence condition for the uniform limit map $f$ to be $\mathcal{F}$-transitive or weakly $\mathcal{F}$-sensitive or $\mathcal{F}$-sensitive or $\left(\mathcal{F}_{1}, \mathcal{F}_{2}\right)$-sensitive and a necessary condition for the uniform limit map $f$ to be weakly $\mathcal{F}$-sensitive or $\mathcal{F}$-sensitive or $\left(\mathcal{F}_{1}, \mathcal{F}_{2}\right)$-sensitive. Our results extend and improve some existing ones. (C)2017 All rights reserved.
\end{abstract}

Keywords: Furstenberg families, transitivity, $\mathcal{F}$-transitivity, sensitivity, weak $\mathcal{F}$-sensitivity, $\mathcal{F}$-sensitivity, $\left(\mathcal{F}_{1}, \mathcal{F}_{2}\right)$-sensitivity. 2010 MSC: 37B10, 37C20, 37C50.

\section{Introduction}

Throughout this paper, a topological dynamical system is a pair $(X, f)$, where $f: X \rightarrow X$ is a continuous surjective map acting on a compact metric space $X$ with a metric $d$, and $\mathbb{Z}^{+}$denotes the set of nonnegative integers.

Finding conditions assuring the preservation of a chaotic property under limit operations is an interesting problem (see $[2,6,7,12,14,17,19])$. In [17] the author proved that if the $f_{n}$ are continuous functions acting on a metric space $(X, d)$ converging uniformly to a function $f$ and $f_{n}$ is topologically transitive for all $n \geqslant 1$, then $f$ is not necessarily topologically transitive, and he gave some sufficient conditions for the uniform limit function $f$ to be topologically transitive. Recently, Fedeli and Donne [7] studied the dynamical behavior of the uniform limit map of a sequence of continuous selfmaps of a compact metric space satisfying (topological) transitivity or other related properties and gave some conditions for the topological transitivity of such a limit map. In [5], the author studied the limit behavior of sequences with the form $f_{n} \circ \cdots \circ f_{1}(x), x \in[0,1]$, and whether the simplicity (respectively chaoticity) of $f$ implies the simplicity (respectively chaoticity) of $f_{1, \infty}$, where $f_{1, \infty}=\left(f_{n}\right)$ is a sequence of continuous interval maps which converges uniformly to a continuous map $f$. More recently, in [6] the author considered nonautonomous discrete dynamical systems $f_{1, \infty}$, which is given by sequence $\left(f_{n}\right)$ of surjective continuous maps $f_{n}: I \rightarrow I$ converging uniformly to a map $f: I \rightarrow I$, where $I=[0,1]$, and showed that, even if the full

\footnotetext{
*Corresponding author

Email addresses: gdoulrs@163.com (Risong Li), datom@189.cn (Yu Zhao), wanghq3333@126.com (Hongqing Wang)
} 
Lebesgue measure of a distributionally scrambled set of the nonautonomous system does not guarantee the existence of distributional chaos of the uniform limit map and conversely, there is a nonautonomous system with arbitrarily small distributionally scrambled set that converges uniformly to a map, which is distributionally chaotic a.e.. It is well-known that the sensitivity property characterizes the unpredictability of chaotic phenomenon and it is the essential condition of various definitions of a system to be chaotic. Therefore, when is a system sensitive? This question has gained some attention in more recent papers (see $[1,4,8,9,15,16])$. More recently, in [19] the authors gave an equivalence conditions for the uniform limit map $f$ to be sensitive.

Roughly speaking, a dynamical system $(X, f)$ is sensitive if for any region $U$ of the phase space, there exist two points in $U$ and an integer $n \geqslant 0$ such that the $n$th iterates of the two points under the map $f$ are significantly separated. For a dynamical system, the size of the set of all $n \in Z^{+}$where this significant separation or sensitivity happens can be thought of as a measure of how sensitive the dynamical system is. Especially, if this set is quite thin with arbitrarily large gaps between consecutive entries, then one has some excuse for treating the dynamical system as practically non-sensitive!

For continuous self-maps of compact metric spaces, Moothathu [16] introduced stronger forms of sensitivity in terms of large subsets of $Z^{+}$. Mainly he considered syndetic sensitivity and cofinite sensitivity. Also, he constructed a topologically transitive and sensitive map which is not syndetically sensitive and proved that the followings hold: (1) any syndetically transitive, non-minimal map is syndetically sensitive (this improves the result that sensitivity is redundant in Devaney's definition of chaos); (2) any sensitive map of $[0,1]$ is cofinitely sensitive; (3) any sensitive subshift of finite type is cofinitely sensitive; (4) any syndetically transitive, infinite subshift is syndetically sensitive; (5) no Sturmian subshift is cofinitely sensitive.

In [12], we obtained an equivalence condition for the uniform limit map $f$ to be topologically transitive or syndetically transitive or topologically weak mixing or topological mixing and a necessary condition for the uniform limit map $f$ to be sensitive or cofinitely sensitive or multi-sensitive. In [14], we gave the correct proofs of Theorems 3.4-3.7 in [12] and presented an equivalence condition for the uniform limit map $f$ to be syndetically sensitive or cofinitely sensitive or multi-sensitive or ergodically sensitive and a sufficient condition for the uniform limit map $f$ to be totally transitive or topologically weak mixing, where a sequence $\left(f_{n}\right)$ of continuous selfmaps of a compact metric space $X$ converging uniformly to a continuous selfmap $f$ of the compact metric space $X$.

In [18], the authors introduced and studied some concepts of sensitivity via Furstenberg families. Moreover, by investigating these notions they obtained some interesting results. In particular, they discussed the sensitivity of symbolic dynamical systems in the sense of Furstenberg families. Inspired by [19] we further study the chaoticity of the uniform limit maps via Furstenberg families.

In this paper, given a sequence $\left(f_{n}\right)$ of continuous selfmaps of a compact metric space $X$ which converges uniformly to a continuous selfmap $f$ of a compact metric space $X$ and three Furstenberg families $\mathcal{F}$, $\mathcal{F}_{1}$ and $\mathcal{F}_{2}$, we establish an equivalence condition for the uniform limit map $f$ to be $\mathcal{F}$-transitive or weakly $\mathcal{F}$-sensitive or $\mathcal{F}$-sensitive or $\left(\mathcal{F}_{1}, \mathcal{F}_{2}\right)$-sensitive and a necessary condition for the uniform limit map $f$ to be weakly $\mathcal{F}$-sensitive or $\mathcal{F}$-sensitive or $\left(\mathcal{F}_{1}, \mathcal{F}_{2}\right)$-sensitive. These results extend and improve some existing ones.

The organization of this paper is as follows: In Section 2, we recall some concepts. Main results are established in Section 3.

\section{Preliminaries}

Firstly we complete some notations and recall some concepts.

A subset $S \subset Z^{+}$is thick if $S$ contains arbitrarily large blocks of consecutive numbers. A subset $S \subset Z^{+}$ is syndetic if $Z^{+} \backslash S$ is not thick.

Let $(X, f)$ be a dynamical system. According to the classical definition, $f$ is sensitive if there exists $\delta>0$ such that for any $x \in X$ and any open neighborhood $V_{x}$ of $x$, there is $n \in Z^{+}$such that $\sup \left\{d\left(f^{n}(x), f^{n}(y)\right)\right.$ : 
$\left.y \in V_{x}\right\}>\delta$. One can write this in a slightly different way. For $V \subset X$ and $\delta>0$, let $N_{f}(V, \delta)=\left\{n \in Z^{+}\right.$: there exist $x, y \in V$ with $d\left(f^{n}(x), f^{n}(y)\right)>\delta$. Now, we say:

(1) $f$ is sensitive if there exists $\delta>0$ such that $N_{f}(V, \delta)$ is nonempty for any nonempty open set $V \subset X$;

(2) $f$ is syndetically sensitive if there is $\delta>0$ such that $N_{f}(V, \delta)$ is syndetic for every nonempty open subset $\mathrm{V} \subset \mathrm{X}$;

(3) $f$ is cofinitely sensitive if there is $\delta>0$ such that $N_{f}(V, \delta)$ is cofinite for every nonempty open subset $V \subset X$, that is, $Z^{+} \backslash N_{f}(V, \delta)$ is finite;

(4) $f$ is multi-sensitive if there is $\delta>0$ such that for every integer $k>0, \bigcap_{i=1}^{k} N_{f}\left(V_{i}, \delta\right) \neq \emptyset$ for any nonempty open subsets $\mathrm{V}_{1}, \mathrm{~V}_{2}, \cdots, \mathrm{V}_{\mathrm{k}} \subset \mathrm{X}$;

(5) $f$ is ergodically sensitive (see $[10,11,13]$ ) if there is $\delta>0$ such that $N_{f}(V, \delta)$ has positive upper density for every nonempty open subset $V \subset X$, that is,

$$
\limsup _{n \rightarrow \infty} \frac{1}{n}\left|N_{f}(V, \delta) \cap\{0,1, \cdots, n-1\}\right|>0,
$$

where $|A|$ denotes the cardinality of $A$.

Clearly, by the definitions syndetic sensitivity implies ergodic sensitivity, and ergodic sensitivity implies sensitivity. Corollary 3 and Theorem 5 from [16] show that every Sturmian subshift is syndetically sensitive, and that no Sturmian subshift is cofinitely sensitive. In addition, Theorem 7 in [16] shows that there exists a sensitive subshift which is not syndetically sensitive. Consequently, there exist sensitive transformations that are not syndetically sensitive, and syndetically sensitive maps that are not cofinitely sensitive.

For any given two dynamical systems $(X, f)$ and $(X, g), d_{\infty}(f, g)=\sup _{x \in X} d(f(x), g(x))$, where $(X, d)$ is a perfect metric space (i.e., $X$ is closed and has no isolated points) (see [17]).

Now we introduce some notations related to a family. Let $\mathbb{Z}^{+}$be the set of all nonnegative integers, and let $\mathcal{P}$ be the collection of all subsets of $\mathbb{Z}^{+}$. A subset $\mathcal{F} \subset \mathcal{P}$ is called a Furstenberg family (see [3]) if it is hereditary upwards, that is, $F_{1} \subset F_{2}$ and $F_{1} \in \mathcal{F}$ imply $F_{2} \in \mathcal{F}$.

Let $\mathcal{F}$ be a Furstenberg family. A dynamical system $(X, f)$ is $\mathcal{F}$-sensitive if there is an $\varepsilon>0$ such that for any given $x \in X$ and any given open neighborhood $U$ of $x$, there is $y \in U$ such that the pair $(x, y)$ is not $\mathcal{F}$ - $\varepsilon$-asymptotic (i.e., $\left.\left\{n: d\left(f^{n}(x), f^{n}(y)\right)>\varepsilon\right\} \in \mathcal{F}\right)$. The $\varepsilon>0$ is called a constant of $\mathcal{F}$-sensitivity for $f$ (see [18]).

Let $\mathcal{F}_{1}$ and $\mathcal{F}_{2}$ be Furstenberg families. A dynamical system $(X, f)$ is $\left(\mathcal{F}_{1}, \mathcal{F}_{2}\right)$-sensitive if there is an $\varepsilon>0$ such that each point $x \in X$ is a limit of points $y \in X$ such that the pair $(x, y)$ is $\mathcal{F}_{1}$-proximal but not $\mathcal{F}_{2}-\varepsilon$-asymptotic (that is, $\left\{\mathrm{n}: \mathrm{d}\left(\mathrm{f}^{\mathrm{n}}(\mathrm{x}), \mathrm{f}^{\mathrm{n}}(\mathrm{y})\right)<\delta\right\} \in \mathcal{F}_{1}$ for any $\delta>0$ and $\left.\left\{\mathrm{n}: \mathrm{d}\left(\mathrm{f}^{\mathrm{n}}(\mathrm{x}), \mathrm{f}^{\mathrm{n}}(\mathrm{y})\right)>\varepsilon\right\} \in \mathcal{F}_{2}\right)$. The $\varepsilon>0$ is called a constant of $\left(\mathcal{F}_{1}, \mathcal{F}_{2}\right)$-sensitivity for $f$ (see [18]).

\section{Main results}

In all theorems it is assumed that $\lim _{n \rightarrow \infty} d_{\infty}\left(f_{n}^{n}, f^{n}\right)=0$.

It is well-known that family machinery is applied to describe the family versions of several stronger forms of sensitivity and transitivity, and different notions can be unified by this family viewpoint. So, this kind of machinery is a very important tool in topological dynamical system and ergodic theory.

Let $\mathcal{F}$ be a Furstenberg family and let $\mathcal{B}$ be the family consisting of all infinite subsets of $\mathbb{Z}^{+}$. Write $N_{f}(U, V)=\left\{n \in \mathbb{Z}^{+}: U \cap f^{-n}(V) \neq \emptyset\right\}$ for any $U, V \subset X$. Clearly, $N_{f}(U, V)=\left\{n \in \mathbb{Z}^{+}: f^{n}(U) \cap V \neq \emptyset\right\}$. A map $f: X \rightarrow X$ of a metric space $X$ is said to be $\mathcal{F}$-transitive if $N_{f}(U, V) \in \mathcal{F}$ for any two nonempty and open sets $\mathrm{U}, \mathrm{V} \subset \mathrm{X}$. By the definitions, we know that for a given continuous map $f: X \rightarrow X$ of a metric space $X$, the followings hold: 
(1) $f$ is topologically transitive if and only if $N_{f}(U, V) \in \mathcal{B}$ for any two nonempty open sets $U, V \subset X$;

(2) $f$ is syndectically transitive if and only if $N_{f}(U, V) \in \mathcal{F}_{s}$ for any two nonempty open sets $U, V \subset X$, where $\mathcal{F}_{s}$ is the collection of all syndetic subsets of $\mathbb{Z}^{+}$;

(3) $f$ is topologically ergodic if and only if $N_{f}(U, V) \in \mathcal{F}_{e}$ for any two nonempty open sets $U, V \subset X$, where $\mathcal{F}_{e}=\left\{A \subset \mathbb{Z}^{+}:\right.$A has positive upper density $\}$;

(4) $f$ is topologically weak mixing if and only if $N_{f}(U, V) \in \mathcal{F}_{t}$ for any two nonempty open sets $U, V \subset X$, where $\mathcal{F}_{t}$ is the collection of all thick subsets of $\mathbb{Z}^{+}$;

(5) $f$ is topologically mixing if and only if $N_{f}(U, V) \in \mathcal{F}_{\text {cof }}$ for any two nonempty open sets $U, V \subset X$, where $\mathcal{F}_{\text {cof }}$ is the collection of all cofinite subsets of $\mathbb{Z}^{+}$;

(6) $f$ is sensitive if and only if there exists $\delta>0$ such that $N_{f}(V, \delta) \in \mathcal{B}$ for any nonempty open set $V \subset X$;

(7) $f$ is syndetically sensitive if and only if there exists $\delta>0$ such that $N_{f}(V, \delta) \in \mathcal{F}_{s}$ for any nonempty open set $\mathrm{V} \subset \mathrm{X}$;

(8) $f$ is cofinitely sensitive if and only if there exists $\delta>0$ such that $N_{f}(V, \delta) \in \mathcal{F}_{\text {cof }}$ for any nonempty open set $V \subset X$;

(9) $f$ is ergodically sensitive if and only if there exists $\delta>0$ such that $N_{f}(V, \delta) \in \mathcal{F}_{e}$ for any nonempty open set $V \subset X$.

For a Furstenberg family $\mathcal{F}$, its dual family is

$$
\kappa \mathcal{F}=\left\{\mathrm{F} \in \mathcal{P}: F \cap F^{\prime} \neq \emptyset, \forall F^{\prime} \in \mathcal{F}\right\} .
$$

It is easily seen that

$$
\kappa \mathcal{F}=\left\{\mathrm{F} \in \mathcal{P}: \mathbb{Z}^{+} \backslash \mathrm{F} \notin \mathcal{F}\right\},
$$

and that if $\mathcal{F}$ is a Furstenberg family then so is $\kappa \mathcal{F}$. A Furstenberg family $\mathcal{F}$ is said to be full if and only if $\mathcal{F} \cdot K \mathcal{F} \subset \mathcal{B}$, where $\mathcal{F} \cdot K \mathcal{F}=\{F \cap G: F \in \mathcal{F}, G \in K \mathcal{F}\}$. By the definition we can see that $\mathcal{B}, \mathcal{F}_{s}, \mathcal{F}_{t}$, and $\mathcal{F}_{\text {cof }}$ are full. Furthermore, it is easily verified that if $\mathcal{F}$ is a given full Furstenberg family, and if $A=\left\{a_{i}: a_{i}<\right.$ $\left.a_{i+1}, i \in\{0,1, \cdots\}\right\} \in \mathcal{F}$, then for any given integer $m \geqslant 0, A^{\prime}=\left\{a_{i}: a_{i}<a_{i+1}, i \in\{m, m+1, \cdots\}\right\} \in \mathcal{F}$.

Theorem 3.1. Let $(\mathrm{X}, \mathrm{d})$ be a metric space and $\mathcal{F}$ be a full family, and let $\left(\mathrm{f}_{\mathrm{n}}\right)$ be a sequence of continuous functions from $\mathrm{X}$ into itself such that $\left(\mathrm{f}_{\mathrm{n}}\right)$ converges uniformly to a function $\mathrm{f}$. Then $\mathrm{f}$ is $\mathcal{F}$-transitive if and only if $\mathrm{N}_{\left(\mathrm{f}_{n}^{\mathfrak{n}}\right)}(\mathrm{U}, \mathrm{V}) \in \mathcal{F}$ for any two nonempty open subsets $\mathrm{U}, \mathrm{V} \in \mathrm{X}$, where

$$
\mathrm{N}_{\left(f_{n}^{\mathfrak{n}}\right)}(\mathrm{U}, \mathrm{V})=\left\{\mathrm{m} \in \mathbb{Z}^{+}: \mathrm{f}_{\mathrm{m}}^{\mathrm{m}}(\mathrm{U}) \cap \mathrm{V} \neq \emptyset\right\} .
$$

Proof. Let $\mathrm{U}, \mathrm{V} \subset \mathrm{X}$ be nonempty and open subsets. Choose $v \in \mathrm{V}$ and $\varepsilon>0$ such that $\mathrm{B}(v, \varepsilon) \subset \mathrm{V}$. By the $\mathcal{F}$-transitivity of $f$ and the assumption that $\lim _{n \rightarrow \infty} d_{\infty}\left(f_{n}^{n}, f^{n}\right)=0$,

and

$$
\mathrm{u} \cap \mathrm{f}^{-\mathrm{n}}\left(\mathrm{B}\left(v, \frac{1}{2} \varepsilon\right)\right) \neq \emptyset
$$

$$
d_{\infty}\left(f_{m}^{m}, f^{m}\right)<\frac{1}{2} \varepsilon,
$$

for some integer $n>0$ and any integer $m \geqslant n$, where

$$
\mathrm{B}\left(v, \frac{1}{2} \varepsilon\right)=\left\{u \in X: d(u, v)<\frac{1}{2} \varepsilon\right\} .
$$


Take

$$
u \in U \cap f^{-n}\left(B\left(v, \frac{1}{2} \varepsilon\right)\right)
$$

Then

$$
d\left(f_{n}^{n}(u), v\right) \leqslant d\left(f_{n}^{n}(u), f^{n}(u)\right)+d\left(f^{n}(u), v\right) \leqslant d_{\infty}\left(f_{n}^{n}, f^{n}\right)+\frac{1}{2} \varepsilon<\varepsilon .
$$

This implies that

$$
f_{n}^{n}(u) \in B(v, \varepsilon) \subset v
$$

Since

$$
(-\infty, m] \cap\left\{n \in \mathbb{N}: U \cap\left(f_{n}\right)^{-n}\left(B\left(v, \frac{1}{2} \varepsilon\right)\right) \neq \emptyset\right\}=(-\infty, m] \cap\left\{n \in \mathbb{N}: f_{n}^{n}(U) \cap B\left(v, \frac{1}{2} \varepsilon\right) \neq \emptyset\right\} \in \mathcal{F},
$$

we have

$$
(-\infty, m] \cap\left\{n \in \mathbb{N}: f_{n}^{n}(U) \cap V \neq \emptyset\right\} \in \mathcal{F}
$$

This means that

$$
\left\{n \in \mathbb{N}: f_{n}^{n}(U) \cap V \neq \emptyset\right\} \in \mathcal{F}
$$

Now, we suppose that

$$
\left\{\mathrm{n} \in \mathbb{N}: f_{\mathfrak{n}}^{\mathfrak{n}}(\mathrm{U}) \cap \mathrm{V} \neq \emptyset\right\} \in \mathcal{F},
$$

for any two nonempty and open subsets $\mathrm{U}, \mathrm{V} \subset \mathrm{X}$. Choose $v \in \mathrm{V}$ and $\varepsilon>0$ with

$$
\mathrm{B}(v, \varepsilon) \subset \mathrm{V} .
$$

Then

$$
\mathrm{U} \cap \mathrm{f}_{\mathrm{n}}^{-\mathrm{n}}\left(\mathrm{B}\left(v, \frac{1}{2} \varepsilon\right)\right) \neq \emptyset \text { and } \mathrm{d}_{\infty}\left(\mathrm{f}_{\mathrm{m}}^{\mathrm{m}}, \mathrm{f}^{\mathrm{m}}\right)<\frac{1}{2} \varepsilon,
$$

for some integer $n>0$ and any integer $m \geqslant n$. Fix

$$
u \in U \cap f_{n}^{-n}\left(B\left(v, \frac{1}{2} \varepsilon\right)\right)
$$

Then we have

$$
d\left(f^{n}(u), v\right) \leqslant d\left(f^{n}(u), f_{n}^{n}(u)\right)+d\left(f_{n}^{n}(u), v\right) \leqslant d_{\infty}\left(f_{n}^{n}, f^{n}\right)+\frac{1}{2} \varepsilon<\varepsilon .
$$

This implies that

$$
f^{n}(u) \in B(v, \varepsilon) \subset V
$$

Since

$$
\begin{gathered}
(-\infty, m] \cap\left\{n \in \mathbb{N}: U \cap f^{-n}\left(B\left(v, \frac{1}{2} \varepsilon\right)\right) \neq \emptyset\right\} \in \mathcal{F}, \\
(-\infty, m] \cap\left\{n \in \mathbb{N}: f^{n}(U) \cap B\left(v, \frac{1}{2} \varepsilon\right) \neq \emptyset\right\} \in \mathcal{F} .
\end{gathered}
$$

This means that

$$
(-\infty, m] \cap\left\{n \in \mathbb{N}: f^{n}(U) \cap V \neq \emptyset\right\} \in \mathcal{F}
$$

Consequently,

$$
\left\{\mathrm{n} \in \mathbb{N}: \mathrm{f}^{\mathrm{n}}(\mathrm{U}) \cap \mathrm{V} \neq \emptyset\right\} \in \mathcal{F} .
$$

By the definition, $f$ is $\mathcal{F}$-transitive. This completes the proof.

Remark 3.2. Theorem 3.1 extends and improves the corresponding results in [14, 19]. 
A dynamical system $(X, f)$ is weakly $\mathcal{F}$-sensitive (see [3]) if there exists some $\varepsilon>0$ (a constant of weak $\mathcal{F}$-sensitivity for $f$ ) such that for any open subset $U \subset X$ there are two points $x, y \in U$ such that the pair $(x, y)$ is not $\mathcal{F}-\varepsilon$-asymptotic, that is,

$$
\left\{n \in \mathbb{Z}^{+}: d\left(f^{n}(x), f^{n}(y)\right)>\varepsilon\right\} \in \mathcal{F} .
$$

Theorem 3.3. Let $(X, d)$ be a metric space and $\mathcal{F}$ be a given full family, and let $\left(f_{n}\right)$ be a sequence of continuous functions from $\mathrm{X}$ into itself such that $\left(\mathrm{f}_{\mathrm{n}}\right)$ converges uniformly to a function $\mathrm{f}$. Then $\mathrm{f}$ is $\mathcal{F}$-sensitive if and only if there is $\delta>0$ such that $\mathrm{N}_{\left(\mathrm{f}_{n}\right)}(\mathrm{V}, \delta) \in \mathcal{F}$ for any nonempty open set $\mathrm{V} \subset \mathrm{X}$, where $\mathrm{N}_{\left(\mathrm{f}_{n}^{\mathfrak{n}}\right)}(\mathrm{V}, \delta)=\left\{\mathrm{n} \in \mathrm{Z}^{+}\right.$: there are $x, y \in V$ with $\left.\mathrm{d}\left(f_{\mathfrak{n}}^{\mathfrak{n}}(x), f_{\mathfrak{n}}^{\mathfrak{n}}(\mathrm{y})\right)>\delta\right\}$.

Proof. Since $\lim _{n \rightarrow \infty} d_{\infty}\left(f_{n}^{n}, f_{n}^{n}\right)=0$, for any $\varepsilon>0$, there is an integer $n_{0}>0$ such that

$$
\left|d\left(f_{n}^{n}(x), f_{n}^{n}(y)\right)-d\left(f^{n}(x), f^{n}(y)\right)\right| \leqslant d\left(f_{n}^{n}(x), f^{n}(x)\right)+d\left(f_{n}^{n}(y), f^{n}(y)\right)<\frac{1}{4} \varepsilon+\frac{1}{4} \varepsilon=\frac{1}{2} \varepsilon,
$$

for any $x, y \in X$ and any integer $n \geqslant n_{0}$.

Now we suppose on the contrary that there is $\delta>0$ such that $N_{\left(f_{n}\right)}(V, \delta) \in \mathcal{F}$ for any nonempty open set $V \subset X$, and that $f$ is not $\mathcal{F}$-sensitive. Then, for every $\varepsilon>0$ and any integer $m \geqslant n_{0}$, there are an integer $k \geqslant m$ with $k \in \mathcal{F}$, an $x \in X$ and a neighborhood $U$ of $x$ such that for all $x^{\prime}, y \in U$,

$$
d\left(f^{k}\left(x^{\prime}\right), f^{k}(y)\right)<\frac{\varepsilon}{2} .
$$

By (3.1),

$$
d\left(f_{k}^{k}\left(x^{\prime}\right), f_{k}^{k}(y)\right)<\varepsilon
$$

for all $x^{\prime}, y \in U$. This is a contradiction.

Assume that $f$ is $\mathcal{F}$-sensitive, and that for any $\delta>0$, there is nonempty open set $\mathrm{V} \subset \mathrm{X}$ such that $N_{\left(f_{n}\right)}(V, \delta) \notin \mathcal{F}$. Then, for any $\varepsilon>0$, there are $x \in X$ and a neighborhood $U$ of $x$ such that $N_{\left(f_{n}^{n}\right)}\left(U, \frac{1}{2} \varepsilon\right) \notin$ $\mathcal{F}$. This implies that for any integer $m \geqslant n_{0}$, there is an integer $k \geqslant m$ with $k \notin N_{\left(f_{n}^{\mathfrak{n}}\right)}\left(U, \frac{1}{2} \varepsilon\right)$. By (3.1),

$$
d\left(f^{k}(x), f^{k}(y)\right)<\varepsilon,
$$

for all $x, y \in U$. It is a contradiction. Thus, the proof is complete.

Remark 3.4. Theorem 3.3 extends and improves the corresponding results in [14, 19].

Theorem 3.5. Let $(X, d)$ be a metric space and $\mathcal{F}$ be a given full family, and let $\left(f_{n}\right)$ be a sequence of continuous functions from $\mathrm{X}$ into itself such that $\left(\mathrm{f}_{\mathrm{n}}\right)$ converges uniformly to a function $\mathrm{f}$. Then $\mathrm{f}$ is weakly $\mathcal{F}$-sensitive if and only if there is $\varepsilon>0$ such that for any nonempty open set $\mathrm{V} \subset \mathrm{X}$, there are two points $\mathrm{x}, \mathrm{y} \in \mathrm{V}$ with $\left\{\mathrm{n} \in \mathrm{Z}^{+}: \mathrm{d}\left(\mathrm{f}_{\mathfrak{n}}^{\mathrm{n}}(\mathrm{x}), \mathrm{f}_{\mathfrak{n}}^{\mathrm{n}}(\mathrm{y})\right)>\varepsilon\right\} \in \mathcal{F}$.

Proof. Now we suppose on the contrary that there is $\varepsilon>0$ such that for any nonempty open set $\mathrm{V} \subset \mathrm{X}$, there are two points $x, y \in V$ with $\left\{n \in Z^{+}: d\left(f_{n}^{n}(x), f_{n}^{n}(y)\right)>\varepsilon\right\} \in \mathcal{F}$, and that $f$ is not weakly $\mathcal{F}$-sensitive. By (3.1), for the above $\varepsilon>0$, we get

$$
\left|d\left(f_{n}^{n}(x), f_{n}^{n}(y)\right)-d\left(f^{n}(x), f^{n}(y)\right)\right| \leqslant d\left(f_{n}^{n}(x), f^{n}(x)\right)+d\left(f_{n}^{n}(y), f^{n}(y)\right)<\frac{1}{4} \varepsilon+\frac{1}{4} \varepsilon=\frac{1}{2} \varepsilon,
$$

for any $x, y \in V$ and any integer $n \geqslant n_{0}$. Then, for the above $\varepsilon>0$, there is a nonempty open set $V \subset X$ such that

for any $x, y \in V$. By (3.2),

$$
\left[\mathrm{n}_{0},+\infty\right) \cap\left\{\mathrm{k} \in \mathbb{Z}^{+}: \mathrm{d}\left(\mathrm{f}^{\mathrm{k}}(\mathrm{x}), \mathrm{f}^{\mathrm{k}}(\mathrm{y})\right)>\frac{3 \varepsilon}{2}\right\} \notin \mathcal{F},
$$

$$
d\left(f_{n}^{\mathfrak{n}}(x), f_{n}^{\mathfrak{n}}(y)\right)>\varepsilon,
$$


for any $x, y \in V$ and any

$$
n \in\left[n_{0},+\infty\right) \cap\left\{k \in \mathbb{Z}^{+}: d\left(f^{k}(x), f^{k}(y)\right)>\frac{3 \varepsilon}{2}\right\} .
$$

This implies that

$$
\left[n_{0},+\infty\right) \cap\left\{k \in \mathbb{Z}^{+}: \mathrm{d}\left(\mathrm{f}_{\mathrm{k}}^{\mathrm{k}}(\mathrm{x}), \mathrm{f}_{\mathrm{k}}^{\mathrm{k}}(\mathrm{y})\right)>\varepsilon\right\} \notin \mathcal{F},
$$

for any $x, y \in V$. So,

$$
\left\{k \in \mathbb{Z}^{+}: d\left(f_{k}^{k}(x), f_{k}^{k}(y)\right)>\varepsilon\right\} \notin \mathcal{F},
$$

for any $x, y \in V$. It is a contradiction.

Assume that $f$ is weakly $\mathcal{F}$-sensitive, and that for any $\varepsilon>0$, there is a nonempty open set $V \subset X$ such that

$$
\left\{k \in \mathbb{Z}^{+}: d\left(f_{k}^{k}(x), f_{k}^{k}(y)\right)>\varepsilon\right\} \notin \mathcal{F},
$$

for any $x, y \in V$. By the definition, there is some $\varepsilon>0$ such that

$$
\left\{k \in \mathbb{Z}^{+}: d\left(f^{k}(x), f^{k}(y)\right)>\frac{3}{2} \varepsilon\right\} \in \mathcal{F},
$$

for some $x, y \in V$. This implies that

$$
\left[n_{0},+\infty\right) \cap\left\{k \in \mathbb{Z}^{+}: d\left(f^{k}(x), f^{k}(y)\right)>\frac{3}{2} \varepsilon\right\} \in \mathcal{F} .
$$

By (3.1),

$$
d\left(f_{k}^{k}(x), f_{k}^{k}(y)\right)>\varepsilon,
$$

for all $x, y \in V$ and any

$$
k \in\left[n_{0},+\infty\right) \cap\left\{k \in \mathbb{Z}^{+}: d\left(f^{k}(x), f^{k}(y)\right)>\frac{3}{2} \varepsilon\right\} .
$$

This means that

$$
\left[n_{0},+\infty\right) \cap\left\{k \in \mathbb{Z}^{+}: d\left(f_{k}^{k}(x), f_{k}^{k}(y)\right)>\varepsilon\right\} \in \mathcal{F},
$$

for all $x, y \in V$. So,

$$
\left\{k \in \mathbb{Z}^{+}: d\left(f_{k}^{k}(x), f_{k}^{k}(y)\right)>\varepsilon\right\} \in \mathcal{F},
$$

for all $x, y \in V$. It is a contradiction. Thus, the proof is complete.

Theorem 3.6. Let $(\mathrm{X}, \mathrm{d})$ be a metric space and $\mathcal{F}_{1}, \mathcal{F}_{2}$ be two given full families, and let $\left(\mathrm{f}_{\mathrm{n}}\right)$ be a sequence of continuous functions from $\mathrm{X}$ into itself such that $\left(\mathrm{f}_{\mathrm{n}}\right)$ converges uniformly to a function $\mathrm{f}$. Then, $\mathrm{f}$ is $\left(\mathcal{F}_{1}, \mathcal{F}_{2}\right)-$ sensitive if and only if there is $\varepsilon>0$ such that each point $x \in X$ is a limit of points $y \in X$ such that the pair $(x, y)$ satisfies that

$$
\left\{n \in Z^{+}: d\left(f_{n}^{n}(x), f_{n}^{n}(y)\right)<\delta\right\} \in \mathcal{F}_{1},
$$

for any $\delta>0$, and that

$$
\left\{n \in Z^{+}: d\left(f_{n}^{\mathfrak{n}}(x), f_{\mathfrak{n}}^{\mathfrak{n}}(y)\right)>\varepsilon\right\} \in \mathcal{F}_{2} .
$$

Proof. Now we suppose that there is $\varepsilon>0$ such that each point $x \in X$ is a limit of points $y \in X$ such that the pair $(x, y)$ satisfies that

$$
\left\{\mathrm{n} \in \mathrm{Z}^{+}: \mathrm{d}\left(\mathrm{f}_{\mathfrak{n}}^{\mathrm{n}}(x), \mathrm{f}_{\mathfrak{n}}^{\mathrm{n}}(\mathrm{y})\right)<\delta\right\} \in \mathcal{F}_{1},
$$

for any $\delta>0$, and that

$$
\left\{n \in Z^{+}: d\left(f_{n}^{n}(x), f_{n}^{n}(y)\right)>\varepsilon\right\} \in \mathcal{F}_{2} .
$$

By (3.1), for the above $\varepsilon>0$, we get

$$
\left|d\left(f_{n}^{n}(x), f_{n}^{n}(y)\right)-d\left(f^{n}(x), f^{n}(y)\right)\right| \leqslant d\left(f_{n}^{n}(x), f^{n}(x)\right)+d\left(f_{n}^{n}(y), f^{n}(y)\right)<\frac{1}{4} \varepsilon+\frac{1}{4} \varepsilon=\frac{1}{2} \varepsilon,
$$


for any $x, y \in X$ and any integer $n \geqslant n_{0}$. So, we have that

$$
d\left(f^{n}(x), f^{n}(y)\right) \geqslant d\left(f_{n}^{n}(x), f_{n}^{n}(y)\right)-\frac{1}{2} \varepsilon,
$$

for any $x, y \in X$ and any integer $n \geqslant n_{0}$. This implies that if a pair $(x, y) \in X \times X$ satisfies that

$$
\left\{n \in Z^{+}: d\left(f_{n}^{n}(x), f_{n}^{n}(y)\right)>\varepsilon\right\} \in \mathcal{F}_{2},
$$

then the pair $(x, y) \in X \times X$ satisfies that

$$
\left\{n \in Z^{+}: d\left(f^{n}(x), f^{n}(y)\right)>\frac{1}{2} \varepsilon\right\} \in \mathcal{F}_{2} .
$$

By (3.1), for any given $\delta>0$, there is an integer $n_{0}>0$ such that

$$
\left|d\left(f_{n}^{n}(x), f_{n}^{n}(y)\right)-d\left(f^{n}(x), f^{n}(y)\right)\right| \leqslant d\left(f_{n}^{n}(x), f^{n}(x)\right)+d\left(f_{n}^{n}(y), f^{n}(y)\right)<\frac{1}{4} \delta+\frac{1}{4} \delta=\frac{1}{2} \delta,
$$

for any $x, y \in X$ and any integer $n \geqslant n_{0}$. Therefore,

$$
d\left(f^{n}(x), f^{n}(y)\right) \leqslant d\left(f_{n}^{n}(x), f_{n}^{n}(y)\right)+\frac{1}{2} \delta
$$

for any $x, y \in X$ and any integer $n \geqslant n_{0}$. This means that if a pair $(x, y) \in X \times X$ satisfies that

$$
\left\{\mathrm{n} \in \mathrm{Z}^{+}: \mathrm{d}\left(\mathrm{f}_{\mathfrak{n}}^{\mathrm{n}}(x), \mathrm{f}_{\mathfrak{n}}^{\mathrm{n}}(\mathrm{y})\right)<\delta\right\} \in \mathcal{F}_{1},
$$

for any $\delta>0$, then the pair $(x, y) \in X \times X$ satisfies that

$$
\left\{n \in Z^{+}: d\left(f^{n}(x), f^{n}(y)\right)<\frac{3}{2} \delta\right\} \in \mathcal{F}_{1}
$$

for any $\delta>0$. By the definition and the above argument, $f$ is $\left(\mathcal{F}_{1}, \mathcal{F}_{2}\right)$-sensitive.

Assume that $f$ is $\left(\mathcal{F}_{1}, \mathcal{F}_{2}\right)$-sensitive. Then, by the definition, there is $\varepsilon>0$ such that each point $x \in X$ is a limit of points $y \in X$ such that the pair $(x, y) \in X \times X$ satisfies that

$$
\left\{n \in Z^{+}: d\left(f^{n}(x), f^{n}(y)\right)<\delta\right\} \in \mathcal{F}_{1},
$$

for any $\delta>0$, and

$$
\left\{n \in Z^{+}: d\left(f^{n}(x), f^{n}(y)\right)>\varepsilon\right\} \in \mathcal{F}_{2} .
$$

By (3.3), for the above $\varepsilon>0$ and any $x, y \in X$, there exists a positive integer $n_{0}$ such that

$$
d\left(f_{n}^{n}(x), f_{n}^{n}(y)\right) \geqslant d\left(f^{n}(x), f^{n}(y)\right)-\frac{1}{2} \varepsilon
$$

for any $x, y \in X$ and any integer $n \geqslant n_{0}$. This implies that if a pair $(x, y) \in X \times X$ satisfies that

$$
\left\{n \in Z^{+}: d\left(f^{n}(x), f^{n}(y)\right)>\varepsilon\right\} \in \mathcal{F}_{2},
$$

then the pair $(x, y) \in X \times X$ satisfies that

$$
\left\{n \in Z^{+}: d\left(f_{n}^{n}(x), f_{n}^{n}(y)\right)>\frac{1}{2} \varepsilon\right\} \in \mathcal{F}_{2} .
$$

By (3.4), for any given $\delta>0$, there is an integer $n_{0}>0$ such that

$$
d\left(f_{n}^{n}(x), f_{n}^{n}(y)\right) \leqslant d\left(f^{n}(x), f^{n}(y)\right)+\frac{1}{2} \delta
$$


for any $x, y \in X$ and any integer $n \geqslant n_{0}$. This means that if a pair $(x, y) \in X \times X$ satisfies that

$$
\left\{n \in Z^{+}: d\left(f^{n}(x), f^{n}(y)\right)<\delta\right\} \in \mathcal{F}_{1},
$$

for any $\delta>0$, then the pair $(x, y) \in X \times X$ satisfies that

$$
\left\{\mathrm{n} \in \mathrm{Z}^{+}: \mathrm{d}\left(\mathrm{f}_{\mathrm{n}}^{\mathrm{n}}(\mathrm{x}), \mathrm{f}_{\mathrm{n}}^{\mathrm{n}}(\mathrm{y})\right)<\frac{3}{2} \delta\right\} \in \mathcal{F}_{1},
$$

for any $\delta>0$. By the above argument, there is $\varepsilon>0$ such that each point $x \in X$ is a limit of points $y \in X$ such that the pair $(x, y) \in X \times X$ satisfies that

$$
\left\{n \in Z^{+}: d\left(f_{n}^{n}(x), f_{n}^{n}(y)\right)<\delta\right\} \in \mathcal{F}_{1},
$$

for any $\delta>0$, and

$$
\left\{n \in Z^{+}: d\left(f_{n}^{n}(x), f_{n}^{n}(y)\right)>\varepsilon\right\} \in \mathcal{F}_{2} .
$$

We are done.

The following lemma which comes from [2] is needed.

Lemma 3.7. Let $(\mathrm{X}, \mathrm{d})$ be a compact metric space, and let $\left(\mathrm{f}_{\mathrm{n}}\right)$ be a sequence of continuous functions from $\mathrm{X}$ into itself such that $\left(f_{n}\right)$ converges uniformly to a function $f$. Then given $\varepsilon>0$ and a positive integer $l$ there exists a positive integer $n_{0}$ (possibly depending on $\mathrm{l}$ ) such that for any integer $\mathrm{n}>\mathrm{n}_{0}, \mathrm{~d}\left(\mathrm{f}_{\mathfrak{n}}^{\mathrm{l}}(\mathrm{x}), \mathrm{f}^{\mathrm{l}}(\mathrm{x})\right)<\varepsilon$ for all $\mathrm{x} \in \mathrm{X}$.

Theorem 3.8. Let $(X, d)$ be a compact metric space and $\mathcal{F}$ be a fixed family, and let $\left(f_{n}\right)$ be a sequence of continuous functions from $\mathrm{X}$ into itself such that $\left(\mathrm{f}_{\mathrm{n}}\right)$ converges uniformly to a function $\mathrm{f}$. If the integer $\mathrm{n}_{0}$ in Lemma 3.7 is independent of $\mathrm{l}$ and $\mathrm{f}$ is $\mathcal{F}$-sensitive with $\delta$ as a constant of sensitivity, then there exists an integer $\mathrm{N}>0$ such that $\mathrm{f}_{\mathrm{n}}$ is $\mathcal{F}$-sensitive with $\frac{1}{3} \delta$ as a constant of sensitivity for any $\mathrm{n} \geqslant \mathrm{N}$.

Proof. Suppose that $f$ is $\mathcal{F}$-sensitive with $\delta>0$ as a constant of $\mathcal{F}$-sensitivity for $f$. Then, by the definition, for any nonempty and open set $V \subset X, N_{f}(V, \delta) \in \mathcal{F}$. By hypothesis and Lemma 3.7, there is an integer $N>0$, which is independent of $l$, such that for any integer $n>N$,

$$
d\left(f_{n}^{l}(x), f^{l}(x)\right)<\frac{1}{3} \delta
$$

for any integer $l \geqslant 1$ and any $x \in X$. This means that

$$
d\left(f_{n}^{j}(x), f^{j}(x)\right)<\frac{1}{3} \delta
$$

for any integer $n>N$, any $j \in N_{f}(V, \delta)$, and any $x \in X$. So, we have that

$$
d\left(f_{n}^{j}(x), f_{n}^{j}(y)\right) \geqslant d\left(f^{j}(x), f^{j}(y)\right)-d\left(f_{n}^{j}(x), f^{j}(x)\right)-d\left(f_{n}^{j}(y), f^{j}(y)\right)>\delta-2 \cdot \frac{1}{3} \delta=\frac{1}{3} \delta,
$$

for any integer $n>N$, any $j \in N_{f}(V, \delta)$ and any $x, y \in V$ with $x \neq y$. This implies that

$$
\mathrm{N}_{\mathrm{f}}(\mathrm{V}, \delta) \subset \mathrm{N}_{\left(\mathrm{f}_{\mathrm{n}}\right)}\left(\mathrm{V}, \frac{1}{3} \delta\right)
$$

for any integer $n>N$. As

$$
\mathrm{N}_{\mathrm{f}}(\mathrm{V}, \delta) \in \mathcal{F}, \quad \mathrm{N}_{\left(\mathrm{f}_{\mathrm{n}}\right)}\left(\mathrm{V}, \frac{1}{3} \delta\right) \in \mathcal{F},
$$

for any integer $n>N$. Thus, the proof is complete.

Remark 3.9. Theorem 3.8 extends and improves the corresponding results in [12]. 
Theorem 3.10. Let $(X, d)$ be a compact metric space and $\mathcal{F}$ be a fixed family, and let $\left(f_{n}\right)$ be a sequence of continuous functions from $X$ into itself such that $\left(f_{n}\right)$ converges uniformly to a function $f$. If the integer $n_{0}$ in Lemma 3.7 is independent of $\mathrm{l}$ and $\mathrm{f}$ is weakly $\mathcal{F}$-sensitive with $\delta$ as a constant of weak $\mathcal{F}$-sensitivity, then there exists an integer $\mathrm{N}>0$ such that $\mathrm{f}_{\mathrm{n}}$ is weakly $\mathcal{F}$-sensitive with $\frac{1}{3} \delta$ as a constant of weak $\mathcal{F}$-sensitivity for any integer $\mathrm{n} \geqslant \mathrm{N}$.

Proof. Suppose that $f$ is weakly $\mathcal{F}$-sensitive with $\delta$ as a constant of weak $\mathcal{F}$-sensitivity. Then, by the definition, for any open subset $U \subset X$ there are two points $x, y \in U$ such that

$$
\left\{n \in \mathbb{Z}^{+}: d\left(f^{n}(x), f^{n}(y)\right)>\delta\right\} \in \mathcal{F} .
$$

By hypothesis and Lemma 3.7, there is an integer $N>0$, which is independent of $l$, such that for any integer $n>N$,

$$
d\left(f_{n}^{l}(x), f^{l}(x)\right)<\frac{1}{3} \delta
$$

for any integer $l \geqslant 1$ and any $x \in X$. This means that

$$
d\left(f_{n}^{j}(x), f^{j}(x)\right)<\frac{1}{3} \delta
$$

for any integer $n>N$, any $j \in\left\{n \in \mathbb{Z}^{+}: d\left(f^{n}(x), f^{n}(y)\right)>\delta\right\}$, and any $x \in X$. So, for the above pair $(x, y)$ we have that

$$
d\left(f_{n}^{j}(x), f_{n}^{j}(y)\right) \geqslant d\left(f^{j}(x), f^{j}(y)\right)-d\left(f_{n}^{j}(x), f^{j}(x)\right)-d\left(f_{n}^{j}(y), f^{j}(y)\right)>\delta-2 \cdot \frac{1}{3} \delta=\frac{1}{3} \delta,
$$

for any integer $n>N$ and any $j \in\left\{n \in \mathbb{Z}^{+}: d\left(f^{n}(x), f^{n}(y)\right)>\delta\right\}$. This implies that

$$
\left\{n \in \mathbb{Z}^{+}: d\left(f^{n}(x), f^{n}(y)\right)>\delta\right\} \subset\left\{j \in \mathbb{Z}^{+}: d\left(f_{n}^{j}(x), f_{n}^{j}(y)\right)>\frac{1}{3} \delta\right\},
$$

for any integer $n>N$. As

$$
\left\{n \in \mathbb{Z}^{+}: d\left(f^{n}(x), f^{n}(y)\right)>\delta\right\} \in \mathcal{F}, \quad\left\{j \in \mathbb{Z}^{+}: d\left(f_{n}^{j}(x), f_{n}^{j}(y)\right)>\frac{1}{3} \delta\right\} \in \mathcal{F},
$$

for any integer $n>N$. By the definition, $f_{n}$ is weakly $\mathcal{F}$-sensitive with $\frac{1}{3} \delta$ as a constant of weak $\mathcal{F}$ sensitivity for any integer $n \geqslant N$. Thus, the proof is complete.

Theorem 3.11. Let $(X, d)$ be a compact metric space and $\mathcal{F}_{1}, \mathcal{F}_{2}$ be two fixed families, and let $\left(f_{n}\right)$ be a sequence of continuous functions from $X$ into itself such that $\left(f_{n}\right)$ converges uniformly to a function $f$. If the integer $n_{0}$ in Lemma 3.7 is independent of $l$ and $f$ is $\left(\mathcal{F}_{1}, \mathcal{F}_{2}\right)$-sensitive with $\delta$ as a constant of $\left(\mathcal{F}_{1}, \mathcal{F}_{2}\right)$-sensitivity, then there exists an integer $\mathrm{N}>0$ such that $\mathrm{f}_{\mathrm{n}}$ is $\left(\mathcal{F}_{1}, \mathcal{F}_{2}\right)$-sensitive with $\frac{1}{3} \delta$ as a constant of $\left(\mathcal{F}_{1}, \mathcal{F}_{2}\right)$-sensitivity for any integer $n \geqslant N$.

Proof. Suppose that $f$ is $\left(\mathcal{F}_{1}, \mathcal{F}_{2}\right)$-sensitive with $\delta$ as a constant of $\left(\mathcal{F}_{1}, \mathcal{F}_{2}\right)$-sensitivity. Then, by hypothesis and the definition, every point $x \in X$ is a limit of points $y \in X$ such that the pair $(x, y) \in X \times X$ satisfies that

$$
\left\{n \in Z^{+}: d\left(f^{n}(x), f^{n}(y)\right)<\varepsilon\right\} \in \mathcal{F}_{1},
$$

for any $\varepsilon>0$, and that

$$
\left\{n \in Z^{+}: d\left(f^{n}(x), f^{n}(y)\right)>\delta\right\} \in \mathcal{F}_{2} .
$$

By hypothesis and Lemma 3.7, there is an integer $N>0$, which is independent of $l$, such that for any integer $n>N$,

$$
d\left(f_{n}^{l}(x), f^{l}(x)\right)<\frac{1}{3} \delta,
$$


for any integer $l \geqslant 1$ and any $x \in X$. This means that

$$
d\left(f_{n}^{j}(x), f^{j}(x)\right)<\frac{1}{3} \delta
$$

for any integer $n>N$, any $j \in\left\{n \in \mathbb{Z}^{+}: d\left(f^{n}(x), f^{n}(y)\right)>\delta\right\}$, and any $x \in X$. So, if the pair $(x, y)$ satisfies that

$$
\left\{n \in Z^{+}: d\left(f^{n}(x), f^{n}(y)\right)>\delta\right\} \in \mathcal{F}_{2}
$$

then we have that

$$
d\left(f_{n}^{j}(x), f_{n}^{j}(y)\right) \geqslant d\left(f^{j}(x), f^{j}(y)\right)-d\left(f_{n}^{j}(x), f^{j}(x)\right)-d\left(f_{n}^{j}(y), f^{j}(y)\right)>\delta-2 \cdot \frac{1}{3} \delta=\frac{1}{3} \delta,
$$

for any integer $n>N$ and any $j \in\left\{n \in \mathbb{Z}^{+}: d\left(f^{n}(x), f^{n}(y)\right)>\delta\right\}$. This implies that

$$
\left\{n \in \mathbb{Z}^{+}: d\left(f^{n}(x), f^{n}(y)\right)>\delta\right\} \subset\left\{j \in \mathbb{Z}^{+}: d\left(f_{n}^{j}(x), f_{n}^{j}(y)\right)>\frac{1}{3} \delta\right\},
$$

for any integer $n>N$. As

$$
\left\{n \in \mathbb{Z}^{+}: d\left(f^{n}(x), f^{n}(y)\right)>\delta\right\} \in \mathcal{F}_{2}, \quad\left\{j \in \mathbb{Z}^{+}: d\left(f_{n}^{j}(x), f_{n}^{j}(y)\right)>\frac{1}{3} \delta\right\} \in \mathcal{F}_{2},
$$

for any integer $n>N$.

By (3.1), for any given $\varepsilon>0$, there is an integer $n_{0}>0$ such that

$$
\left|d\left(f_{n}^{n}(x), f_{n}^{n}(y)\right)-d\left(f^{n}(x), f^{n}(y)\right)\right| \leqslant d\left(f_{n}^{n}(x), f^{n}(x)\right)+d\left(f_{n}^{n}(y), f^{n}(y)\right)<\frac{1}{4} \varepsilon+\frac{1}{4} \varepsilon=\frac{1}{2} \varepsilon,
$$

for any $x, y \in X$ and any integer $n \geqslant n_{0}$. Therefore,

$$
d\left(f_{n}^{n}(x), f_{n}^{n}(y)\right) \leqslant d\left(f^{n}(x), f^{n}(y)\right)+\frac{1}{2} \varepsilon,
$$

for any $x, y \in X$ and any integer $n \geqslant n_{0}$. This means that if a pair $(x, y) \in X \times X$ satisfies that

$$
\left\{n \in Z^{+}: d\left(f^{n}(x), f^{n}(y)\right)<\varepsilon\right\} \in \mathcal{F}_{1},
$$

for any $\varepsilon>0$, then the pair $(x, y) \in X \times X$ satisfies that

$$
\left\{j \in Z^{+}: d\left(f_{\mathfrak{n}}^{j}(x), f_{\mathfrak{n}}^{j}(y)\right)<\frac{3}{2} \varepsilon\right\} \in \mathcal{F}_{1},
$$

for any $n \geqslant n_{0}$. By the definition and the above argument, $f_{n}$ is $\left(\mathcal{F}_{1}, \mathcal{F}_{2}\right)$-sensitive for any integer $n \geqslant$ $\max \left\{n_{0}, N\right\}$. Thus, the proof is complete.

\section{Acknowledgment}

The authors are very grateful to the referees for their careful reading, comments, and suggestions, which help us improve this paper.

This research was supported by the Project of Enhancing School With Innovation of Guangdong Ocean University (Grant NO. GDOU2016050207), the Key Scientific and Technological Research Project of Science and Technology Department of Zhanjiang City (Grant 2010C3112005), the National Natural Science Foundation of China (11501391), the Opening Project of Artificial Intelligence Key Laboratory of Sichuan Province (2015RZJ01) and the Opening Project of Bridge Non-destruction Detecting and Engineering Computing Key Laboratory of Sichuan Province (2014QZJ02). 


\section{References}

[1] C. Abraham, G. Biau, B. Cadre, Chaotic properties of mappings on a probability space, J. Math. Anal. Appl., 266 (2002), 420-431. 1

[2] R. Abu-Saris, K. Al-Hami, Uniform convergence and chaotic behavior, Nonlinear Anal., 65 (2006), 933-937. 1, 3

[3] E. Akin, Recurrence in topological dynamics. Furstenberg families and Ellis actions, The University Series in Mathematics, Plenum Press, New York, (1997). 2, 3

[4] J. Banks, J. Brooks, G. Cairns, G. Davis, P. Stacey, On Devaney's definition of chaos, Amer. Math. Monthly, 99 (1992), 332-334. 1

[5] J. S. Cánovas, Li-Yorke chaos in a class of nonautonomous discrete systems, J. Difference Equ. Appl., 17 (2011), $479-486$. 1

[6] J. Dvořáková, Chaos in nonautonomous discrete dynamical systems, Commun. Nonlinear Sci. Numer. Simul., 17 (2012), 4649-4652. 1

[7] A. Fedeli, A. Le Donne, A note on the uniform limit of transitive dynamical systems, Bull. Belg. Math. Soc. Simon Stevin, 16 (2009), 59-66. 1

[8] E. Glasner, B. Weiss, Sensitive dependence on initial conditions, Nonlinearity, 6 (1993), 1067-1075. 1

[9] L.-F. He, X.-H. Yan, L.-S. Wang, Weak-mixing implies sensitive dependence, J. Math. Anal. Appl., 299 (2004), $300-304$. 1

[10] R.-S. Li, A note on shadowing with chain transitivity, Commun. Nonlinear Sci. Numer. Simul., 17 (2012), $2815-2823$. 5

[11] R.-S. Li, A note on stronger forms of sensitivity for dynamical systems, Chaos Solitons Fractals, 45 (2012), 753-758. 5

[12] R.-S. Li, A note on uniform convergence and transitivity, Chaos Solitons Fractals, 45 (2012), 759-764. 1, 3.9

[13] R.-S. Li, The large deviations theorem and ergodic sensitivity, Commun. Nonlinear Sci. Numer. Simul., 18 (2013), 819-825. 5

[14] R.-S. Li, H.-Q. Wang, Erratnm to "A note on uniform convergence and transitivity [Chaos, Solitons and Fractals 45 (2012), 759-764]", Chaos Solitons Fractals, 59 (2014), 112-118. 1, 3.2, 3.4

[15] H. Liu, E.-H. Shi, G.-F. Liao, Sensitivity of set-valued discrete systems, Nonlinear Anal., 71 (2009), 6122-6125. 1

[16] T. K. S. Moothathu, Stronger forms of sensitivity for dynamical systems, Nonlinearity, 20 (2007), 2115-2126. 1, 2

[17] H. Román-Flores, Uniform convergence and transitivity, Chaos Solitons Fractals, 38 (2008), 148-153. 1, 2

[18] H.-Y. Wang, J.-C. Xiong, F. Tan, Furstenberg families and sensitivity, Discrete Dyn. Nat. Soc., 2010 (2010), 12 pages. 1,2

[19] K.-S. Yan, F.-P. Zeng, G.-R. Zhang, Devaney's chaos on uniform limit maps, Chaos Solitons Fractalss, 44 (2011), 522-525. 1, 3.2, 3.4 